\title{
Regeneration in the central nervous system and related factors. Summary of the Bermuda Paraplegia Conference, April 1994 (International Spinal Research Trust)
}

\author{
S Davies ${ }^{1}$, L S Illis ${ }^{2}$ and G Raisman ${ }^{3}$ \\ ${ }^{1,3}$ Medical Research Council, National Institute for Medical Research, Mill Hill, London; ${ }^{2}$ Wessex Neurological \\ Centre, Southampton University Hospitals, Southampton
}

Keywords: central nervous system; spinal cord injury researches; regeneration in the central nervous system

\section{Introduction}

The Bermuda Paraplegia Conferences began in the late 1970 s and were held regularly every $2-3$ years. The last two conferences have been organised by the International Spinal Research Trust. The conference consists of a small invited group of scientists and a few clinicians concerned with paraplegia, and the format is that of a workshop.

The conference emphasised experimental work. In this summary we attempt to put the exciting ideas in a more clinical context.

It is worth looking at the way ideas have changed over the last 20 years. The repeated demonstration that the central nervous system (CNS) failed to regenerate had produced a disillusionment amongst neuroscientists. It was not very long ago that any scientist who asked questions about CNS regeneration risked his reputation.

Fortunately some scientists never stopped asking such questions and the answers have been illuminating. In the 1950s and 1960s, sprouting of intact fibres following partial denervation of the CNS was demonstrated, and has since been recognised as a widespread phenomenon. However, although the fact of sprouting is unquestioned, its significance remains uncertain. Is it regeneration and an attempt by the CNS to restore normal function, or is it a random response resulting in haphazard and inappropriate connectivity, or is it a combination of both? Is it initiated by the lesion, or is it a natural and continuing process in the normal CNS?

In the 1970s a new and important field of CNS plasticity was opened up: the unmasking of synapses, indicating the process by which inputs, which in the intact animal produced no response, responded following deafferentation. These experimental findings of sprouting and unmasking have had major implications for the rehabilitation of neurological deficit in man. Together with work on catecholamine agonists and antagonists, the picture arises of an insult to the CNS producing its effect, the clinical syndrome, in two distinct but interrelated ways. On the one hand, there is obvious damage to the CNS, with some recovery at the interface with normal tissue due to reversible damage and circulatory and metabolic changes. This together with secondary pathological effects, perhaps mediated by the release of excitatory amino acids, eventually produces the 'fixed' lesion and fixed deficit of classical and conventional neurology. The production of the fixed lesion is what we were (and unfortunately still are) taught at medical school: the clinical syndrome relates directly to the damaged area of CNS on a simple cause-and-effect system, narrowing the search for the basis of recovery to the site of the lesion and, not surprisingly, producing decades of abortive research.

On the other hand, however, there is an undoubted and as yet unexplained process of recovery after a CNS lesion. This cannot relate directly to the original insult, and must be due to some functional abnormality of the areas of CNS which are necessary for the expression or performance of the behaviour lost as a result of the lesion. This should be seen in the context of reorganisation through sprouting of new synapses and the unmasking of existing synapses, together with their physiological and pharmacological correlates. This concept is beginning to alter the way that clinicians now look at the evolution of a clinical neurological syndrome. In 1963 the demonstration that the synaptic zone may be profoundly altered by the denervation of a critical number of boutons termineaux, with the consequences not only of degeneration of those boutons which had been denervated but also reversible disorganisation of the whole synaptic zone, was advanced as a theory to explain spinal shock. The application of experimental studies to clinical neurological practice indicates (perhaps for the first time) a working theory to suggest an explanation for recovery and for the rationale for neuromodulation techniques in patients with neurological deficit as opposed to pain. The intact, but altered, CNS is theoretically amenable to treatment by externally applied functional electrical systems or by the direct application of neurotransmitters or other substances through an epidural catheter and pump.

In this context, the clinical research delineating the degree of damage in human spinal cord injury, and the 
precise study of complete, incomplete and discomplete lesions is of great importance. It is also of importance for scientists studying regeneration because of the likely implications for both growth and target contact in discomplete versus complete lesions.

Why the emphasis on sprouting, unmasking, the nature of the lesion etc? The reason is simply because all future therapeutic advances must be applied to a spinal cord which is damaged and where the intact CNS has begun to alter or may even be in a chronic static state. In this respect, the human situation is unlikely to resemble the experimental animal model.

In the experimental field the main interests continue in prevention of glial scars, the role of glia in inhibition of growth, regeneration of axons and the study of nerve growth factors. To a lesser extent there is continuing research on the use of bridges and cellular grafts.

This is a summary of the most recent conference held in April 1994.

\section{Human pathology}

Dr Byron Kakulas (Australian Neuromuscular Research Institute, Royal Perth Hospital, Western Australia), presented observations from 494 cases of spinal cord trauma, consisting of 306 'hyperacute' cases with victim dead on arrival at hospital, 113 'acute' survivors of less than 6 months, and 75 'chronic' spinal cord injury survivors of 6 months or longer. A feature of the 'hyperacute' material is the large number of patients with severe vertebral injuries and an intact spinal cord (112 out of 306 cases dead on arrival). Most of these patients survived for less than $1 \mathrm{~h}$ and spinal cord transection was rare. Of the 113 'acute' injuries with fractures, the spine was transected in 13 , and of the 75 'chronic' survivors only 21 showed spinal cord transection.

At first the spinal cord is swollen, bruised and soft with central haemorrhagic necrosis. Axonal damage can be detected within hours and is usually focal. Activated astrocytes appear in 5 or 6 days when glial scar formation begins. Typically a proportion of the white matter of the periphery of the cord is preserved. By 3-6 months after injury, a central multilocular cavity is formed and in the 'chronic' stage the lesion consists of multilocular cysts with gliotic walls, regenerating nerve roots, and a variable residuum of white matter running in the walls of the cavity with collagenous scar tissue. Wallerian degeneration occurs distal (motor) and proximal (sensory) to the lesion. Segmental root atrophy occurs and some evidence of regeneration of nerve roots appears several months after injury. Late complications of spinal cord injury are post-traumatic syringomyelia (3\%), spinal cord ischaemia, and stenosis secondary to osteoarthrosis.

Traumatic demyelination although well documented in experimental studies is not commonly found in human material and true demyelination with preservation of axis cylinders and loss of myelin sheath was only clearly demonstrated in two out of the 24 acute cases.
Remyelination by Schwann cells was seen in two out of 26 chronic cases.

The concept of 'discomplete' lesions was first introduced by Dimitrijevic to explain the electrophysiological transmission of impulses in patients who were clinically complete. Kakulas has extended this term to include neuropathological cases: of the 113 acute SCI patients, 23 were classified as 'discomplete' with no neurological function below the level of the lesion yet with anatomical continuity of the spinal cord at the level of the injury. Of the 75 chronic SCI patients, 28 were discomplete, 20 incomplete, 20 complete and six were unknown.

A large proportion of patients, therefore, have anatomical continuity of the spinal cord at the level of the lesion (the incomplete and discomplete groups) and this emphasises the relative sparing of the white matter even in the most severe injuries and provides a basis for the restorative neurologist to re-establish lost function.

In the incomplete patients the amount of preserved white matter did not directly correlate with function and it seems that the location and quality of the preserved white matter may be more important than quantity in determining the clinical status of the SCI patient. Apart from this important finding there is usually good correlation between the gross neuropathological findings and the neurological state of the patient.

Dr Richard Bunge (Miami Project, USA) gave further detailed information about the pathology of spinal cord injury. He described the findings in acute traumatic central cord syndrome and correlated the magnetic resonance image appearance with the gross pathological and histological features. The primary finding was diffuse disruption of axons, particularly within the lateral columns of the cervical cord in the region occupied by the corticospinal tracts. The central grey matter was intact. The conclusion was that the acute traumatic central cord syndrome is predominantly a white matter injury and that intramedullary haemorrhage is not a necessary feature of the syndrome and, in fact, is probably an uncommon event. $\mathrm{He}$ suggested that in the cervical spinal cord direct compression may occur by buckling of the ligamentum flavum into an already narrowed cervical spinal canal.

Dr Bunge produced data from clinical material and classified four overall categories of spinal cord injury: two categories in which the cord is lacerated or massively compressed and the injury invokes a prominent connective tissue response, and two categories in which the overall cord configuration is retained, the spinal cord surface is not substantially breached, and the connective tissue component of the response minimal. These latter cases involved those in which contusion leads to damage in the central region of the cord with tissue softening and haemorrhage evolving to formation of central fluid filled cysts, and cases in which the substance of the cord is not softened and the intramedullary haemorrhage is not present. He termed the latter type of injury 'solid cord injury'. He described myelin loss in one case. He emphasised that whereas 
demyelination after cord compression is well known in experimental lesions in animals, this appeared to be the first reported case of substantial demyelination following chronic spinal cord compression in humans. Kakulas had earlier commented on this discrepancy between experimental studies and human material.

The detailed descriptions by $\mathrm{Dr}$ Kakulas and $\mathrm{Dr}$ Bunge emphasise the paucity of well documented descriptions of histological changes after spinal cord injury in man and clearly this is relevant in the development of experimental spinal cord injury models.

\section{Damage limitation}

Dr Wise Young (New York University Medical Centre, USA) described a recent multicentre trial of early intervention in spinal cord injury.

The second National Acute Spinal Cord Injury Study (NASCIS 2) showed that high dose methylprednisolone (MP) significantly improves neurological recovery in human spinal cord injury. MP restored $22 \%$ and $75 \%$ of motor scores compared to $8 \%$ and $59 \%$ in placebotreated plegic and paretic patients, respectively. The study, however, raised many questions. Is the dose optimal? Must high drug levels be maintained? How long is the treatment time window? What is the optimal duration? Are treatment parameters the same for all spinal cord injury severities?

Systematic assessment of treatment parameters is a difficult undertaking. Assessing three treatment doses, three times, and three durations at three injury levels, combined with and compared with $\mathrm{MP}$, requires thousands of experiments. No laboratory can carry out such a tour de force. To resolve this problem, eight leading SCI centres have joined in a multicentre animal spinal cord injury study (MASCIC), using the same standardised model and outcome measures to study one treatment completely per year.

Methylprednisolone has a bell-shaped doseresponse curve that depends on injury severity. Severe injuries require more MP over $24 \mathrm{~h}$. One dose shortly after injury is most effective for mild injuries. The monosialic ganglioside GM1 blocked neuroprotective effects of MP. Finally, cyclosporin significantly reduces $24 \mathrm{~h}$ spinal cord lesion volumes but the drug effects depend partially on age and sex.

Multicentre studies will help speed identification of new and better treatments for spinal cord injury. A multicentre study not only provides much greater credibility but allows multiple centres to share expertise and resources for model development and validation of outcome measures. A multicentre approach is essential not only for acute but will improve efficiency of chronic spinal cord injury studies as well.

\section{The distal segment}

Dr L S Illis (Wessex Neurological Centre, Southampton University Hospitals, UK) reviewed events in the distal (spinal) segment - formation of new connections, 'unmasking' and abnormal sensitivity.

The effect of these events is that the intact nervous system has altered structurally and rather than losing its ability to function, the distal segment is reacting in a changed fashion. The more the distal segment reorganises after the original lesion, the more difficult it will be to change. Injecting new information, say, in terms of regeneration of axons or new afferent information may transform the situation. The outcome may be improvement in spasticity, pain, bladder control or movement but it may be a change for the worse with increase in pain or spasticity.

Failure to appreciate changes in the distal segment may condemn excellent research in terms of regrowth of axons to a fascinating intellectual exercise with no practical application.

Can the change in the distal segment actually be investigated in man? The answer is that we can record segmental reflex activity, long tract sensory and motor responses, peripheral and central blood flow and urodynamics and we can, therefore, give a picture of the physiological state of the distal segment and everything which may depend upon it. It is imperative that this is done so that we have a standard model in man ready for testing therapeutic agents which will undoubtedly be available in the near future.

Finally, it is important to remember that the functional effect of a lesion in the spinal cord depends on the segmental level of the lesion (Dr Hans Frankel, Stoke Mandeville, UK). Altering the level by even one segment may have a profound effect on function. For example, a lesion at $\mathrm{C} 4$ or above means that the patient is dependent for feeding, turning, lifting, washing, dressing, bowels, urinal, transfers etc, but a lesion at C5 means that the patient is dependent only for bowels and urinal. A lesion from high thoracic downwards leaves the person fully independent for all personal needs.

\section{Acute response of the CNS to injury}

Dr Hugh Perry (Department of Pharmacology, University of Oxford) showed activation of hippocampal microglia by either excitotoxic cell death or local injection of lipopolysaccharide. There is both proliferation of resident microglia and recruitment of circulating microglia across an intact blood-1brain barrier. The blood-brain barrier breaks down later, and this is associated with neutrophil infiltration. The recruitment of macrophages into the CNS is delayed in comparison to other tissues, has a higher threshold, and is restricted to macrophages.

Dr Solon Thanos (Department of Ophthalmology, University of Tubingen) described the microglial cell phagocytosis of retinal ganglion cells dying after axotomy by lesion of the optic nerve. Retrograde labelling of retinal ganglion cells by carbocyanine and other fluorescent dyes is taken up into the microglia that phagocytose the dead neurons. The label is still present 
in resident retinal microglia (detected after photoconversion to an electron dense product) 3 months after cell death. The microglia immediately in contact with the neuronal somata and dendrites become labelled. The labelled microglia remain resident in the retina. Double labelling shows that microglial cells labelled by normal (developmental) apoptosis acquire a second label by phagocytosis of retinal ganglion cells dying after axotomy in the adult. Microglia become activated at $3 \mathrm{~h}$, and the dead cells are phagocytosed by $24 \mathrm{~h}$ (before the neurons become overtly lytic/ necrotic). Microglial inhibitors (proteases or a tripeptide sequence from human IgG) cause rounding up and retraction of microglial processes and depress microglial migration and phagocytosis. Such microglial inhibition causes longer survival of axotomised retinal ganglion cells (equal to the survival effect of adding growth factors).

Dr Jobst Sievers (Department of Anatomy, University of Kiev) described how microglia, macrophages or circulating monocytes turn into process bearing OX42 positive microglia when seeded on to layers of astrocytes, but not when seeded on to fibroblasts or liver cells. Microglia on astrocytes, or astrocyte conditioned medium induce the formation of inward rectifying potassium channels characteristic of microglia in vivo. Growing dorsal root ganglion axon growth cones collapse on contact with macrophages or deflect from them.

Dr Jerry Silver (Department of Neurosciences Case Western Reserve University) demonstrated cartilagetype proteoglycans in those parts of the embryonic CNS through which axons do not grow and in the dorsal root entry zone where regeneration fails in the adult. As a model of inducing the active state in astrocytes, beta amyloid present in astrocytic zone around senile plaques was plated on dishes. Astrocytes grown on this express proteoglycan and do not allow the vast majority of neurons to attach to this substrate. The occasional neuron that does attach, interestingly grows short processes that tightly encircle the cell body.

The inhibitory effect can be demonstrated to reside in the matrix left behind by the cells, and the area is abrogated by chondroitinase treatment.

Neuronal growth cones confronted in culture with an 'activated' astrocytic process are induced to turn and grow in parallel with that process without growth cone collapse. Monitoring of the uptake of radioactively labelled sulphur by activated astrocytes, suggests that the upregulation of proteoglycan expression is restricted to only one or two subtypes and varies in intensity with different regions of brain and spinal cord.

Dr Ann Logan (Wolfson Research Laboratories, University of Birmingham) reported that transforming growth factor beta (a cytokine involved in inducing fibrosis) and its message appear in a stab wound in cerebral hemisphere. Neutralising antibody (or decorin, a chondroitin sulphate proteoglycan which is a naturally occurring TGF beta antagonist), or the recombinant peptide injected through a lateral ventricular cannula, respectively attenuate or enhance fibrous scar formation and formation of limiting glial membrane.

However RT97 neurofilament and GAP 43 staining of cut axons showed that decorin or neutralising antibody treatment alone failed to permit axonal regeneration across the lesion site.

\section{Strategies for neurite outgrowth}

Professor Clifford Woolf (Department of Anatomy and Developmental Biology, University College, London) used GAP-43 immunoreactivity to demonstrate that peripheral nerve lesions induce the uncut central branches of the dorsal root ganglion neurons to sprout into lamina II to which they do not normally project. Only $20 \%$ of cut central branches of the dorsal root regenerate, and this is not significantly improved by attaching a peripheral nerve graft. Cutting the peripheral branch of the nerve increases the capacity of the dorsal root ganglion to regrow its cut centrally directed branch and induces upregulation of GAP-43. Gap-43 is present in the corticospinal tract, and also in that subgroup of axons that is recruited into a peripheral nerve graft inserted into the thalamus. Professor Woolf suggests that a permissive environment is not sufficient alone for long distance axonal growth and that the intrinsic regenerative growth potential of a cut adult axon must be boosted.

Dr Mellitta Schachner (Department of Neurobiology, Swiss Federal Institute of Technology) pointed out that the glycoprotein L1 is a homophilic binding molecule of the IgG superfamily, expressed on neurons, glia and Schwann cells, and involved in fasciculation of axons and myelination. Transgenic mice expressing L1 under the GFAP (astrocyte specific) promoter overexpress L1 on astrocytes. Both immunohistochemistry and Western blot show that lesions of the optic nerve induce increased GFAP and the transgenic L1. Neurons grown in cryostat sections from the lesioned optic nerve of transgenics expressing L1 at different levels showed enhanced growth of neurites to a degree correlated with the level of L1 expression and decreased by treatment with L1 antibodies. Transgenic L1 was chick specific (unique antibody, acting on only one of the homophilic partners).

L1 has a carbohydrate component identified by the antibody L2/NHK-1 (also expressed on NCAM, J1, and MAG adhesion molecules). L2 positive islands in the motor components of the sciatic nerve may be the cue for regenerating motor axons to find their pathways. In vitro L2 causes a $50 \%$ increase in motor neurite outgrowth, inhibited by anti-L2 antibody; sensory neurites do not respond. Peripheral nerve Schwann cells can remember whether they were specifically motor, and re-express L1.

Dr James Fawcett (Department of Physiology, University of Cambridge) showed that a line of Schwannoma cells secreting basic FGF extruded as a bridge across a knife lesion of the nigrostriate tract caused recovery of rotation in experimental Parkinsonism. He 
grew dorsal root ganglion cells on GFAP-expressing astrocytic cell lines, showing greatest effectiveness in three-dimensional culture. He found no specific phenotypic differences associated with neurite growth permissive cell lines (eg all produced laminin), except that non-permissive lines made more extracellular matrix in aggregate cultures. When the cells were lysed off, the matrix 'footprint' and the conditioned medium had the same permissiveness for neurite outgrowth as the parent cells. The key difference for permissiveness was proteoglycans. A heat-stable factor can block neurite promoting effect of laminin, and is sensitive to enzymes destroying proteoglycans. The non-permissive effect of the Neu7 cell line is destroyed by chlorate (and other reagents) which remove charges from proteoglycan.

Cytoskeletal differences between embryonic and adult axons may explain the failure of adult axons to regenerate. MAP1b (MAP5) is expressed in olfactory neurons and frog retinal ganglion cells, but not in the adult mammalian CNS. The MAP1x subtype is present only in embryonic axons.

Urokinase (which proteolyses extracellular matrix) is produced by invasive cells, and transfecting urokinase into non-permissive cell lines makes them more permissive.

\section{Inhibitory molecules}

Dr Martin Schwab (Brain Research Institute, University of Zurich) described inhibitory proteins specifically produced by myelinating oligodendrocytes which cause long lasting growth cone collapse in culture. Collapse is associated with increased intracellular calcium, and is prevented by drugs that prevent this increase.

The developmental roles of the inhibitory molecule are in late forming tracts, collateralisation, and restriction of axons to specific laminae. After a lesion of the corticospinal tract, treatment by antibodies of the inhibitory molecule increases the distance of axon regeneration after a mid-thoracic lesion in 1-2 month old rats. NT-3, but not BDNF, increases sprouting (measured quantitatively) at the lesion site. NGF has only marginal effects. Trkc (the NT-3 receptor) is present on corticospinal axons, and NT-3 increases the length of elongation induced by antibodies to the inhibitory molecule.

Other fibres, such as serotonergic or adrenergic spinal axons, septo-hippocampal or optic axons also show a growth response to the antibody to the inhibitory molecule.

In behavioural studies with Bregman, ink was used to trace hindlimb footprints, and stride angle and length. The antibody to the inhibitory molecule normalises the stride length after a spinal cord lesion, but not the accuracy of foot placing on a grid, although the contact placing reflex is normalised.

Dr John Steeves (Department of Zoology and Anatomy, University of British Columbia) used a double retrograde label tracing system to show that the long descending tracts of the embryonic chick spinal cord will regenerate if cut before E13, but not after.
Myelination begins at E13. Some functional recovery could be demonstrated by electrical stimulation of reticular formation in decerebrate chickens. Transient suppression of myelination by local intraspinal infusion of anti-myelin antibodies and complement allows anatomical and functional repair, and this is better during embryonic life than after hatching.

\section{Receptors}

Dr Jim Cohen (Department of Anatomy and Cell Biology, Guy's Hospital) provided evidence that receptors for inhibitory molecules do not appear until axons mature. Dorsal root ganglion cells were cultured on cryostat sections of intact or denervated peripheral nerves. Both embryonic and adult cells grow neurites (detected by GAP-43 immunohistochemistry) on denervated nerve. Embryonic, but not adult dorsal root ganglion cell neurites grow well on optic nerve. However the neurites of adult DRG and 10 day postnatal retinal ganglion cells also grew poorly on embryonic optic nerve which contains no mature oligodendrocytes.

\section{Growth factors}

Dr Ron Lindsay (Regeneron Pharmaceuticals, New York) described combinations of growth factors with different temporal aspects of treatment, and the differential effects of different neurotrophin species.

Ciliary neurotrophic factor $(\mathrm{CNF})$ could ameliorate the motor deficits in wobbler mice mutants, and attenuate myofibre atrophy by $50 \%$. Brain-derived neurotrophic factor (BDNF) is similar to CNTF, but less potent. Affected motorneurons lose choline acetyltransferase activity, upregulate p75, and later die. BDNF administered systemically or into the nerve completely prevents choline acetyltransferase loss in adult motorneurons, and alternate daily administration of BDNF and CNTF completely prevents loss of grip strength in wobbler mice.

Dr Yves Barde (Max-Planck Institute for Psychiatry, Munich) studied the effect of neurotrophic factors on developmental cell death in avian embryonic dorsal root and nodose ganglia. Evolutionarily, BDNF seems to be the oldest gene and, according to Dr Barde, nerve growth factor (NGF) developed from it about 600 million years ago to control cell numbers during development. Application of monoclonal antibodies to NT-3 and NGF each cause loss of about one third of the cells-additive loss with both. Anti-NGF specifically knocks out the projections to layer II on the substantia gelatinosa.

The earliest dorsal root fibres to grow in project longitudinally, both rostrally and caudally over several segments, and anti-NT-3 antibody cause complete loss of these.

Knockout of the trkC receptor for NT-3 prevents development of the 1a afferent fibre proprioceptors. 


\section{Refinement of connection during development}

Dr Dale Purves (Department of Neurobiology, Duke University Medical Centre) noted that human brains continue to grow until 20 years of age. There is a steady increase in the total number of brain synapses in the rhesus monkey until puberty. The main function is to store experience.

In the rat primary somatosensory cortex, about 200 barrels represent the body surface. The area is doubled during development, but the parts of the cortex representing the most important sensors grow most, so that the barrel areas become a progressively larger proportion, and the density of the vasculature over the barrels is double that over the inter-barrel regions.

Bilateral eye enucleation at birth augments the size of the somatosensory cortex, and its activity (measured by blood vessel density, deoxyglucose uptake, or electrical recording) is increased. Dr Purves proposed that regeneration of the mammalian CNS may require a substantial modification of its phenotype, which is geared towards information storage and utilisation rather than a regenerative capacity.

$\mathrm{Dr}$ Dennis O'Leary (Molecular Neurobiology Laboratory, Salk Institute, La Jolla) noted that layer 5 cells are the only subcortically projecting cortical cells. The motor cortex projects to the spinal cord and other areas, but not to the superior colliculus. The visual cortex projects to the superior colliculus but not to the spinal cord. The formation of growth cones plays only an early role in the formation of the output rojection from the cortex, not in the later events. nitially the axons grow to all targets, and then ielectively eliminate the inappropriate connections. The primary axons grow through the cerebral peduncle Ind into the spinal dorsal funiculus. Collaterals to the sasilar pontine nuclei are formed later.

In the culture of thick slices, axon branches were ormed over a period of $1 \mathrm{~h}$, de novo from the axon, with no sign of the growth cone. Active sites of ormation tend to be localised to varicosities, which nove up and down overlying the basilar pons, emitting lighly active, transient filopodia. This type of activity nly occurs in the part of the axon overlying the pons.

Culture in a three-dimensional collagen matrix (with iumsden) suggests that the basilar pons produces a diffusible factor initiating activity, ie a tropic axon growth response.

$\mathrm{X}$-irradiation of the E16-18 embryo kills dividing basilar pontine cells, and results in tiny multiple areas of ectopic basilar pontine tissue, against which the zorticospinal axons sent collateral branches which sontacted MAP2 positive dendrites emerging from the jons.

In the spinal cord, layer 5 corticospinal axons also grow past their targets, and then later drop off collaterals to them. The initial map is not somatotopically correct, only later becoming refined. Polysialic acid (PSA) is present in chains on neural cell adhesion molecule (NCAM), removed by the neuraminidase, endo-N, which disrupts the pattern of motor nerves. PSA comes up rapidly over the first few postnatal days
Regeneration in the central nervous system

in the rat corticospinal tract, and injections of endo- $\mathrm{N}$ into the ventricle in the postnatal period (while the corticospinal tract is growing) remove PSA from the corticospinal axons for 10 days, and prevents these axons forming side branches.

Professor Ray Lund (Department of Anatomy, University of Cambridge) described how retino-tectal projections formed by embryonic retinal tissue transplanted over the dorsal midbrain of newborn rats innervate the correct targets but do not make a topographically correct map on the superior colliculus. The pupillary light reflex (which requires only a minimal fibre pathway to the pretectal nucleus) provokes constriction of the pupil to a degree related to the intensity of the projection. Both the projections from the transplants and from the host's own eye can give this response. The animals can learn a light reflex via the transplant. The transfer of information from the transplant to the pupil is possible but not very good. Dark preference behaviour in open field can be driven by the transplant projections but only if the animals are trained.

Fibres from transplants placed on the side of the midbrain also run forward and cross in the optic chiasma even in anophthalmic mutant mice hosts that have never had an eye. Transplants placed in the cerebral peduncle can project to the lateral geniculate nucleus. Transplants to the cortex give a transient outgrowth along the cortical surface, which persists only if there is a co-graft of target tissue.

The superior colliculus may produce BDNF, and the retinal ganglion cells express trkb high affinity receptor. Axons grow out from transplants into litter pockets of BDNF producing fibroblasts co-grafted around them.

In a marsupial mouse which has no eye at birth (the retinal axons reach the chiasm at 7 days, and the superior colliculus at 25-30 days), retinae transplanted before the development of the host visual projections only grow to the tectum after it has become mature.

\section{Re-innervation of target cells in the adult}

Dr Paul Reier (Department of Neurological Surgery, University of Florida College of Medicine) described the transplantation of embryonic spinal cord tissue into contusion lesions of the adult spinal cord. The grafts were anatomically well incorporated. Although there was no reconstruction of long fibre connections, both rats and cats show a recovery of motor function below the lesion. This was attributed to the reconstruction of 'bridge' type circuitry, in which the graft neurons received fibres from the host, and in turn projected to the host spinal cord.

Dr Geoffrey Raisman (Laboratory of Neurobiology, National Institute for Medical Research) showed that the cellular framework of the adult central white matter is permissive to the rapid and profuse growth of axons from transplanted embryonic neurons. These axons elongate for considerable distances, are integrated with the host tract fibres and glia and can reach terminal 
areas of neuropil. The long interfascicular axon growth does not require a correct matching of donor neuron type to host tract but the tract cytoarchitecture defines the pattern of growth with the axons also respecting inter-tract boundaries.

Cultured Schwann cells injected as a suspension become transformed into threadlike cells, migrate and are incorporated into the glial meshwork of both the descending corticospinal tract and the ascending sensory dorsal column tracts of adult host spinal cord.

In long term (untreated) lesions, where the astrocytic scar has become dense, the cut corticospinal axons persist and maintain profuse local sprouting. When cultured Schwann cells are present the sprouting is more intense, and leads to major branching, formation of varicose-bearing arborisations, and elongated sprouts.

Dr Maria Fitzgerald (Department of Anatomy and Embryology, University College, London) discussed the development of the primary spinal afferents into the spinal cord and the competition and rearrangement of the $\mathrm{A}$ and $\mathrm{C}$ fibre innervation of the substantia gelatinosa. This plastic rearrangement occurs in the absence of the still developing GABinergic cortical inhibitory input. Dr Fitzgerald argued a similarity between the neonate spinal cord and a lesioned adult cord in this respect. The developing spinal cord is designed to augment the comparatively limited input it receives compared to a normal adult and in the same way a few regenerated fibres after an adult lesion could elicit a substantial functional recovery. Dr Fitzgerald further highlighted the importance of postsynaptic activation by the regenerating axons for the long term survival and stability of connections.

Dr Rasminsky (Neurosciences Unit, Montreal General Hospital and McGill University) presented experiments in which the optic nerve of adult hamsters was transected within the orbit and an autologous peripheral nerve graft inserted to permit and direct regeneration of retinal ganglion cell (RGC) axons back to the ipsilateral superior colliculus (SC). Extracellular recordings in the SC 15-18 weeks after grafting revealed excitatory and inhibitory postsynaptic responses to visual stimulation which could be blocked with a GABA infusion. At best the regenerated axons could re-innervate $25 \%$ of the available terminal field (mostly with superficial layers) with individual RGC axons making synapses with many SC neurons. The regenerated RGC axons retained a statistically significant correct topographic re-innervation pattern of the SC (roughly by a factor of $3 / 2$ ) with no topographic organisation present within the graft.

\section{Expression of introduced genes}

Professor Gage (Department of Neurosciences, University of California, San Diego) outlined his group's work of bridging the lesioned cholinergic septal hippocampal pathway with fibroblasts, embedded in a collagen matrix and genetically engineered to secrete NGF. The transplants exhibited an initial ingrowth of host astrocytic processes across the host/graft interface and it is these processes that seemed the preferred substrate for the ingrowth and transfer of regenerating cholinergic fibres through the graft into the denervated dentate gyrus. Electron microscopy of AChE staining of the regenerated axons' terminal field formation showed a correct mapping of their synapses on the host dentate gyrus dendrites.

Staining the grafts for NCAM showed a strong correlation with the GFAP+ astrocytes and axon neurofilament staining. The grafts were also strongly nerve growth factor-inducible large external glycoprotein (NILE) positive (a molecule analogous to L1 in mice) and once again this was associated with the neurofilament positive axons. Denervation of the hippocampus causes a transitory upregulation of its NGF levels, possibly acting as a tropic influence on the regenerating axons. However the graft is producing even higher NGF levels suggesting that other factors effect the entry of the regenerating axons into the hippocampus.

Dr Breakefield (Molecular Neurogenetics Unit, Massachusetts General Hospital) reviewed the variety of approaches currently available for transfection. These included:

1. Direct injection of constructs into post mitototic cells (which needs a promoter specific for the target cells).

2. Retroviral construct transfection of mitotic target cells in vivo.

3. Ex vivo; infect cells in culture and then transplant back to proposed site of action.

The third option - with repeated rounds of transfection and clonal selection-achieves high levels of construct integration. Dr Breakefield reported that rat type 1 astrocytes genetically modified in culture via a retroviral mediated gene transfer could constitutively express a mouse $\beta$ NGF transgene producing 40 -fold higher levels of NGF over control astrocytes. Interstriatal co-grafts of these atrocytes with adrenal chromaffin cells increased striatal NGF content approximately $23 \%$ at 4 weeks post transplantation. This was paralleled by a significant reduction of apomorphine induced rotational behaviour in striatal $60 \mathrm{HDA}$ lesioned rats. Astrocytes transfected with a human prepro BDNF cDNA secreted high levels of BDNF giving a 15-fold increase survival of E17 rat retinal ganglion cells (RGCs) grown in these astrocytes conditioned medium.

A number of workers now consider that transplants of genetically engineered astrocytes could provide important methods for delivering a variety of factors influencing neuronal survival and axonal regeneration in neurodegenerative diseases and CNS traumatic injury.

\section{Conclusion}

The field of CNS regeneration is now one of the most exciting aspects of neuroscience. How can anyone fail 
to be optimistic over an unfolding story which includes the demonstration that central axons in contact with Schwann cells regenerate; the identification of molecules on the surface of oligodendrocytes which inhibit the growth of axons; the raising of antibodies to the inhibitory molecules; the generation of the source of antibodies; and the profuse growth of transplanted embryonic neurons.

It is too early to look forward to the clinical implementation of the impressive work summarized here? The improvements expected will be in terms of movement, spasticity, bladder control, pain etc and these are measurable. Unless a model of physiological and clinical assessment is established beforehand and generally accepted, then any such improvement is

unlikely to be taken seriously in the scientific world (see for example the early work on transplantation in Parkinson's disease). The initial effect of applying therapy to a spinal injured person may well be an increase in spasticity or an increase in pain and this must be monitored. Potentially disastrous effects could occur as regards cardiovascular reflexes, and problems relating to abnormalities of bone and muscle. Before any specific therapy can be applied in man there must be a period of training and this must be standardised and generally accepted and the effects of training by itself must be monitored.

The very fact that such questions are now being asked is a tribute to the amount of outstanding work which is summarized in this conference. 